\title{
The Analysis of Alfvén Wave Antenna Implementation in the ETE Spherical Tokamak
}

\author{
L. F. Ruchko and R. M. O. Galvão \\ Institute of Physics, University of São Paulo, \\ CEP 05315-970, São Paulo, SP, Brazil
}

Received on 2 February, 2004; revised version received on 29 April, 2004

\begin{abstract}
The preliminary design study of the Alfvén wave system for the ETE spherical tokamak is presented. The main objective of the proposed $R F$ system is generation of travelling Alfvén waves, which can be used for the study of the non-inductive current drive and the plasma heating in a wide range of operational regimes, which are specific for tokamaks with small aspect ratio $R / a \simeq 1.2-1.5$. The antenna system consists of four modules, which are separated by $90^{\circ}$ in toroidal direction. Each module has two sets of $R F$ current carrying poloidal straps that are positioned symmetrically in relation to the equatorial plane of the vacuum chamber at the poloidal coordinates $\pm 45^{\circ}$. The poloidal extension of each strap is approximately $90^{\circ}$. The excited mode numbers can be controlled by the phasing of the feeding $R F$ current. In the basic regime of operation, the straps in the same toroidal cross-sections are fed by the RF currents with $(0, \pm \pi / 2)$ phasing so that they can excite travelling modes $M= \pm 1, N= \pm 1$. In order to decrease the periphery $R F$ power deposition and to improve the selectivity of the Alfvén mode excitation, the inclination of the antenna straps in toroidal direction can be adjusted in the range of angles $\pm 30^{\circ}$. The dependence of the antenna efficiency on the antenna orientation is studied numerically in the frame of 1-D MHD model.
\end{abstract}

\section{Introduction}

The non-inductive current drive is of crucial importance to spherical tokamaks (ST). A reliable, totally non-inductive plasma current initiation and ramp-up would enable the ST option devoid of inductive transformer coils. Besides, even in present-generation machines the inductive transformer imposes a constraint on the discharge duration and current amplitude. The development of an efficient current drive method for ST is therefore very important.

The ST plasmas generally have very high dielectric constants. These features manifest themselves in the plasma resonance regions becoming almost or completely inaccessible for the electron cyclotron resonance (ECR) and lower hybrid $(\mathrm{LH})$ frequency range waves launched from the outboard plasma periphery. In the low-frequency ion cyclotron resonance (ICR) range high harmonic fast wave (HHFW) can propagate to the core of high temperature, high density plasmas, and damp on electrons, and is therefore can be used for current drive in the ST plasmas. In the initial experiments, which have been carried out on the NSTX device [1] with the antenna phases to launch a toroidally directed spectrum, differences in loop voltage have been observed consistent with plasma current being driven by the wave. The value of the current driven, inferred from the magnetic measurements, is $\sim 100 \mathrm{kA}$ and is roughly consistent with theoretical estimates.

Yet another method of the plasma heating and noninductive current drive, which has no severe plasma density restrictions, is related to the Alfvén wave (AW) excitation. The possibility of utilization of low frequency $\omega<\omega_{c i}$ AWs (here $\omega_{c i}=Z e B / m_{i}$ is the ion cyclotron frequency, $m_{i}$ and $B$ are ion mass and the magnetic field with toroidal component $B_{T}$ and poloidal component $B_{P}$, respectively) for the non-inductive current drive in STs was analyzed in Ref. [2]. The obtained results of numerical calculations have demonstrated efficient generation of significant ponderomotive forces and $R F$ driven currents in STs by the modeconversion of fast waves to kinetic Alfvén waves (KAWs) and their subsequent deposition via combined kinetic and collisional effects. These results are rather optimistic and justify the experimental verification. In the sequel we present the design study of the $R F$ system for the ETE spherical tokamak, which can be used in the experiments with travelling AW excitation in the wide range of plasma parameters.

The RF system of the ETE tokamak was designed with the objective to ensure both effective plasma heating and non-inductive current drive.

It consists of the following parts:

1. Antenna system for excitation of travelling waves with well defined spatial spectrum, which delivers RF energy to the definite plasma zone;

2. Four phase RF generator with output $R F$ power up to $\widetilde{P}_{R F} \approx 1 \mathrm{MW}$

3. RF control and diagnostic system.

The required spatial spectrum is formed by proper phasing of the $R F$ feeding currents. The impedance matching of the antenna with the four-phase oscillator is accomplished by resonant circuits, which form one tuned assembly unit with the RF feeders. The characteristics of the antenna system design with respect to the antenna-plasma coupling and plasma wave excitation for different phasing of the feeding currents are summarized. 


\section{Theoretical considerations}

The principle of the AW heating and current drive scheme is based on the conversion of an externally driven RF field below the ion-cyclotron frequency $\omega_{c i}$, which are launched by the antennae from the plasma boundary, into the KAWs, which are dissipated via combined kinetic and collisional effects. This conversion occurs in inhomogeneous plasmas in the regions, where the $R F$ generator frequency $\omega_{R F}$ matches with the local AW frequency, $k_{\|}(r) C_{A}(r)$. Here $k_{\|}(r)$ is the wave number locally parallel to the ambient magnetic field $\vec{B}, C_{A}(r)$ is the local Alfvén velocity $C_{A}(r)=B / \sqrt{\mu_{0} m_{i} n_{i}(r)}, m_{i}$ and $n_{i}$ are ion mass and number density, respectively. The main details of the mode conversion process can be analyzed in the plane geometry [3]. It was shown that the absorption rate is independent of the dissipation mechanism and remains the same as the result of the magnetohydrodynamic calculation, if the converted wave dissipates completely inside the plasma.

Another basic question, which appears during the AW antenna system design, is choosing the $R F$ generator frequency and the spatial spectrum of the excited modes. The coupling of the antennae to the AW in the plasma is most efficient when the driving frequency is close to the frequency of the natural mode of the plasma-vacuum system. In the AW heating and current drive applications this corresponds to the lowest frequency mode of the fast wave. The presence of the AW conversion zone in the plasma strongly damps this mode, which is called "surface wave", so that there is a broad frequency range over which the efficient coupling is possible.

The theoretical analysis of the AW plasma heating and current drive in tokamaks has been carried out mainly in the cylindrical approximation $[4,5,6]$, which is justified in the case of tokamaks with large aspect ratio $R / a$ (here $R$ and $a$ are the major and minor radius of the plasma column correspondingly). Nevertheless, some features of the AW excitation in tokamaks can be explained only by the toroidal wave coupling $[7,8,9]$. In this case the position of local AW resonances and the dissipation profile in tokamaks can be found with two-fluid toroidal code ALTOK [10]. The problem becomes even more complicated in the case of the spherical tokamaks, when the AW spectrum can be modified both by the toroidal mode coupling and by the noncircular plasma column cross section [11].

Since our main goal is to make a preliminary study of the AW antenna system implementation in the ETE spherical tokamak, and we are interested mainly in choosing the antenna configuration and the generator frequency range, the numerical calculations were made with the help of 1-D cylindrical code, which was described in the Ref.[12]. The plasma column characteristics were simulated by one dimensional MHD model of a current carrying plasma column with finite $\omega / \omega_{c i}$, which was described in details in the Ref.[13]. The effect of antenna feeders was taken into account in the same manner, which was used in the Ref.[14].

The frequency of the $R F$ generator depends on the radial position of the mode conversion zone $r_{A}$ and the excited wave mode numbers $M, N$ according to the dispersion rela- tion

$$
\omega_{R F}^{2}=k_{\| \mid}^{2}\left(r_{A}\right) C_{A}^{2}\left(r_{A}\right)\left(1-\omega_{R F}^{2} / \omega_{c i}^{2}\right),
$$

where

$$
k_{\|}(r)=\frac{N}{R}\left[1+\frac{M}{N q^{*}(r)}\right]
$$

Here we have used the effective safety factor $q^{*}$, which was introduced in the MHD stability studies of the spherical tokamaks $[15,16], q^{*}(r)=\frac{r B_{T}}{R B_{P}(r)}\left(\frac{1+K^{2}}{2}\right), K$ is the vertical elongation, $R$ is the major radius of the plasma column, and $r$ is the radial coordinate of a pseudo-toroidal coordinate system centered at the magnetic axis, $M$ and $N$ are poloidal and toroidal wave numbers correspondingly.

The numerical simulation have been made for the following ETE parameters: $R_{0}=0.3 \mathrm{~m}, a=0.2 \mathrm{~m}, B=0.4 \mathrm{~T}$, $I_{P}=0.2 \mathrm{MA}, n_{0}=3 \times 10^{19} \mathrm{~m}^{-3}, K=1.6$. The equations for $E_{\perp}$ and $B_{\|}$, which determine excitation of fast Alfvén wave and its resonant absorption due to plasma inhomogeneity, are solved for plasma column with parabolic density profile $n=n_{0}\left[(1-\delta)\left(1-r^{2} / a^{2}\right)+\delta\right]$, where a small density jump $n_{0} \delta\left(\delta=10^{-2} ; 10^{-3}\right)$ at the plasma boundary is introduced in order to match boundary conditions and to avoid computational difficulties. Since the antenna system is designed for excitation of fast Alfvén waves, this jump has no influence on calculated characteristics of antenna performance. The plasma current density is assumed to be of the form $j=j_{0}\left(1-r^{2} / a^{2}\right)$. The antenna impedance $Z_{M, N}^{a}$ in the course of the excitation of the AW with mode numbers $M, N$ was determined by the relation $Z_{M, N}^{a}=2 P_{M, N} / I_{a}^{2}$, where $P_{M, N}$ is the $R F$ power input through the mode $M, N$, and $I_{a}$ is the antenna current.

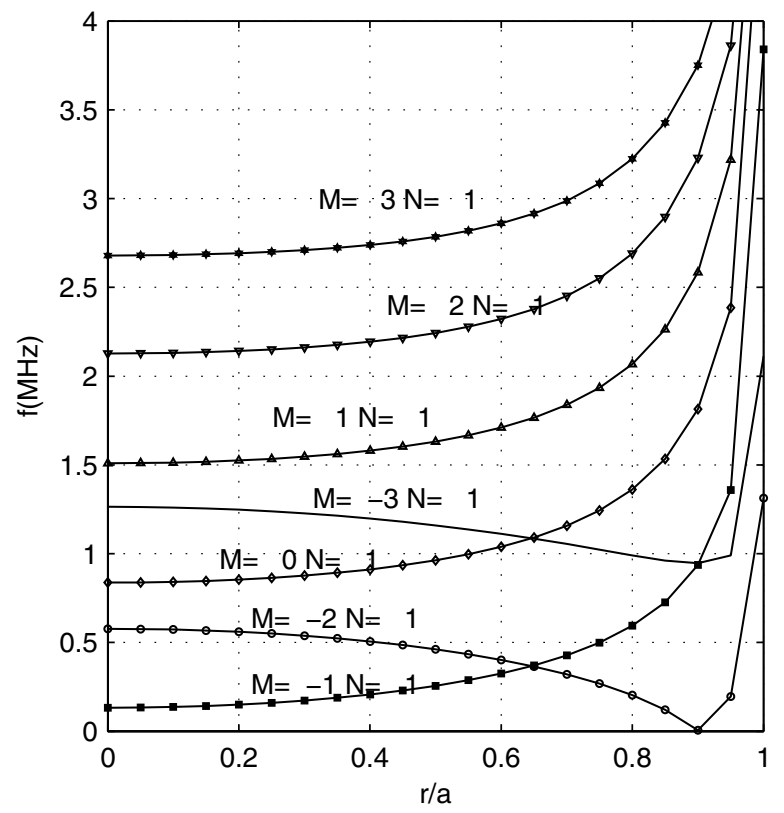

Figure 1. Dependence of the AW frequency on the radial position of the resonance zone for the AW modes with toroidal mode number $N=1$. 


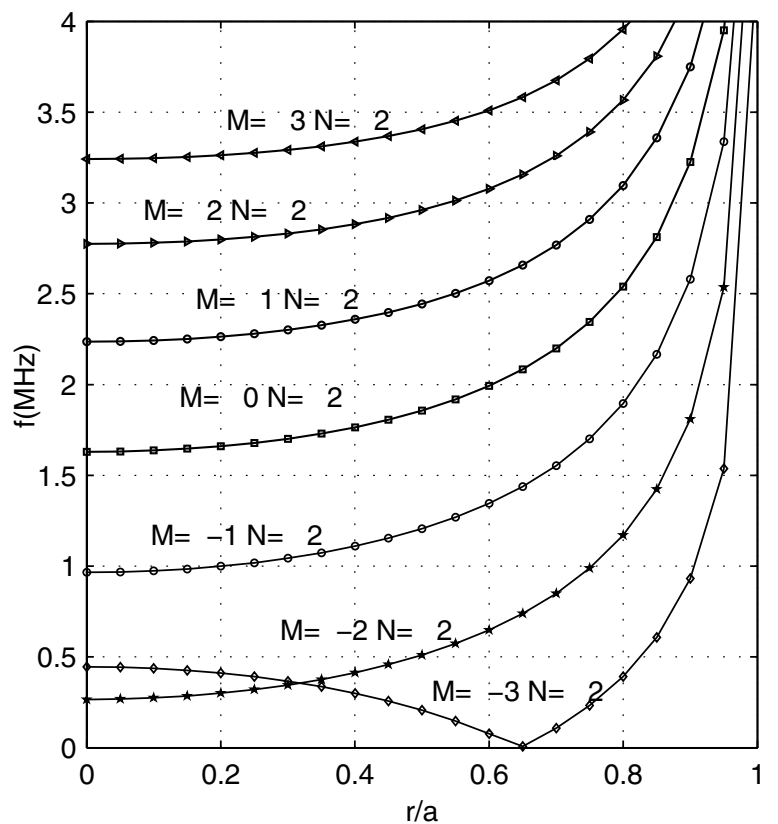

Figure 2. Dependence of the AW frequency on the radial position of the resonance zone for the AW modes with toroidal mode number $N=2$.

In the AW heating scheme, the waves are launched by an external antenna structure, which can define the toroidal wave number $N$. However, because of toroidal mode coupling, the poloidal wave number $M$ is not uniquely defined by the antenna structure. This feature will result in the simultaneous $R F$ power deposition at different radial positions. The calculations of the RF generator frequency for toroidal modes $N=1$ and $N=2$ are shown in Figs. 1, 2 correspondingly.

It is seen that many AW resonance zones can exist for given RF generator frequency at the same time, and in this case $R F$ power deposition zone can not be localized in the predefined plasma region. These calculations lead us to the conclusion that in order to decrease this effect the generator frequency has to be chosen as low as possible. The second conclusion, which can be made, is the need to guarantee the formation of monochromatic toroidal mode spectrum. The results of the AW antenna impedance calculations are shown in Fig. 3.

It is seen that the magnitude of the antenna impedance for the modes of interest is different, and this permits to find the optimal conditions for the $R F$ system operation.

\section{Antenna system of the ETE}

\subsection{Antenna structure}

The design of the AW antenna system for the ETE tokamak has been done with regard to the already existing construction of the vacuum chamber and the necessity to ensure the antenna mounting without major modifications of the existing equipment. On the other hand the excitation of the tra- velling waves has to be guaranteed. In this case the excitation of the monochromatic toroidal spectrum in the ETE tokamak with reasonable number of antenna straps can be accomplished with the eight antenna modules, which are installed in the four toroidal cross-sections of the vacuum chamber. The schematic representation of the proposed antenna structure is shown in Fig. 4 with the corresponding phasing of the $R F$ feeding currents.

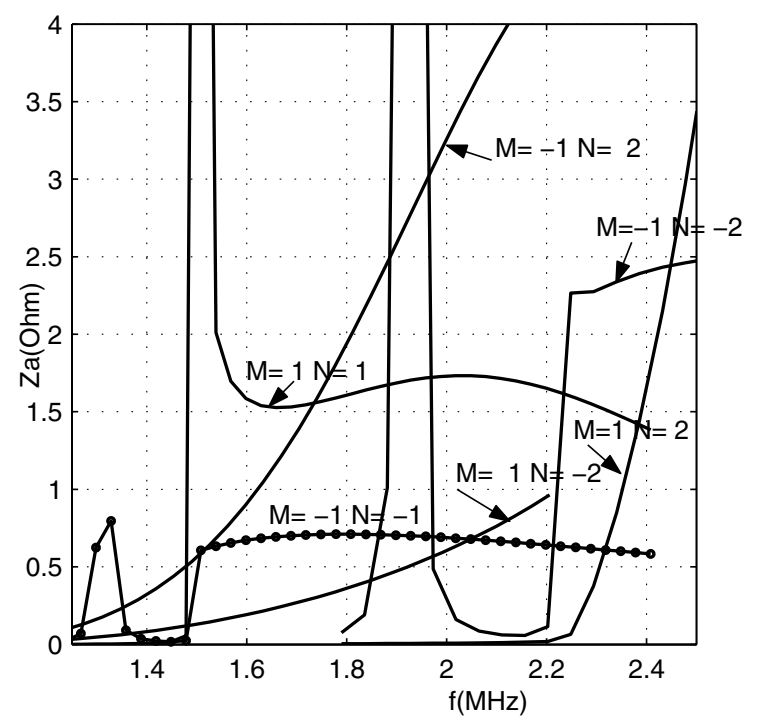

Figure 3. Dependence of the impedance of ideal poloidal antenna on radial Alfvén zone position for different Alfvén modes.

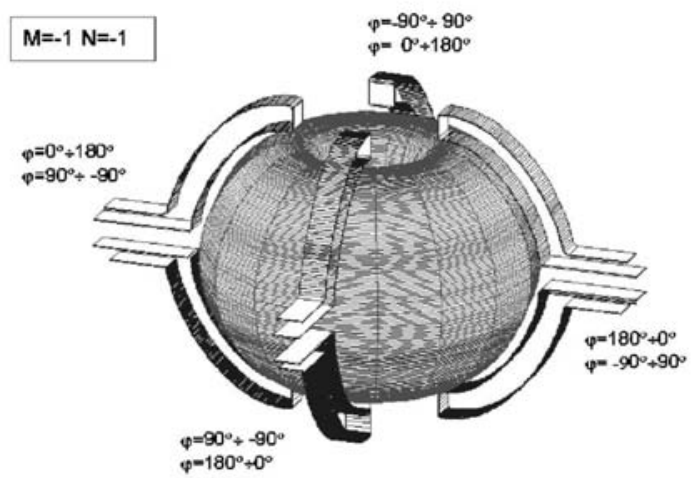

Figure 4. Scheme of antenna straps positioning in the ETE and the phasing of the RF feeding currents for excitation of the mode $\mathrm{M}=-1, \mathrm{~N}=-1$.

The basic component of the antenna system for the ETE spherical tokamak is the strap winding, which has the angular extension in poloidal direction $\varphi \simeq 90^{\circ}$. There are two 
such sets of windings in four toroidal cross-sections of the vacuum chamber of the ETE. Such configuration of an antenna system permits to excite the predominating travelling Alfvén mode with $M= \pm 1, N= \pm 1$. The direction of the wave propagation can be controlled by the corresponding phasing of the antenna feeding currents.

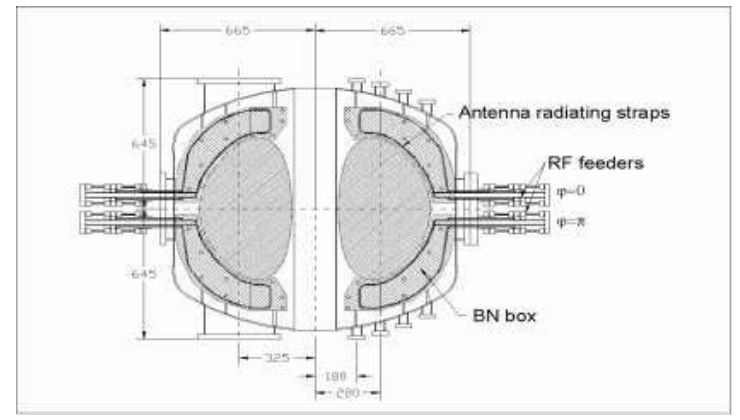

Figure 5. Schematical presentation of antenna construction for the ETE tokamak

The positioning of the antenna elements in the vacuum chamber of the ETE is shown schematically in Fig. 5. The design of the RF system for the ETE is based on model experiments that have been carried out in the TCABR tokamak. The antenna modules are mounted on the vacuum flanges. The mounting of the antenna elements inside the vacuum chamber can be accomplished through the vacuum ports. The construction of antennae ensures protection from the direct antenna-plasma interaction in the form of side and front-end BN protectors. The four-phase RF generator with output power up to $1 \mathrm{MW}$ powers the antennae through the impedance matching units.

\subsection{The impedance spectral characteristics of the antenna system}

The matching of the $R F$ power generator with the antenna load requires the knowledge of antenna parameters in the working range of the operational parameters of the ETE tokamak. In the low frequency range of $R F$ system operation ( $f=1-3 \mathrm{MHz}$ ) the antenna electrical scheme can be represented by the complex impedance $Z=Z_{a}+i Z_{r}$, where $Z_{a}$ is responsible for the $R F$ power dissipation both in the plasma and in the antenna strap itself, and $Z_{r}$ is formed by the antenna strap self inductance and by the inductance introduced by the coupling with the AW plasma oscillations. In the sequel we present the calculations of the part of the antenna impedance, which results from the AW dissipation in the plasma.

The procedure for the antenna impedance calculations consists of the following steps:

- representation of the real antenna geometry as a sum of basic current carrying straps;

- decomposition of the basic elementary current in helical Fourier series ;
- finding field solutions of the boundary value problem for antenna currents, including also the corresponding radial feeding currents;

- summing up the field harmonics with corresponding weight and finding the antenna impedances both for the single $M, N$ mode excitation and for the total antenna delivered power.

The geometry of a basic current carrying antenna element is shown in Fig. 6.

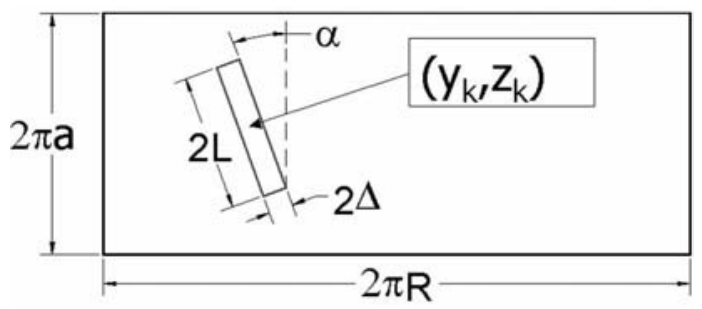

Figure 6. Scheme of one strap antenna current Fourier decomposition.

Since the total antenna impedance is formed by the contributions from the different AW modes, which ensure $R F$ power input in the different plasma regions, we can analyze the conditions of the AW modes excitation in the different regimes of the AW system operation. The spectral contributions to the antenna module impedance, which is fed by the RF current at the frequency $f=2.3 \mathrm{MHz}$, are shown in Fig. 7. It is seen that a rather wide spectrum of the AW modes is excited. The main contribution to the antenna impedance is made by the mode $M=-1, N=-1$.

One module ne $=3.0 \quad \mathrm{f}=2.30 \mathrm{MHz}$

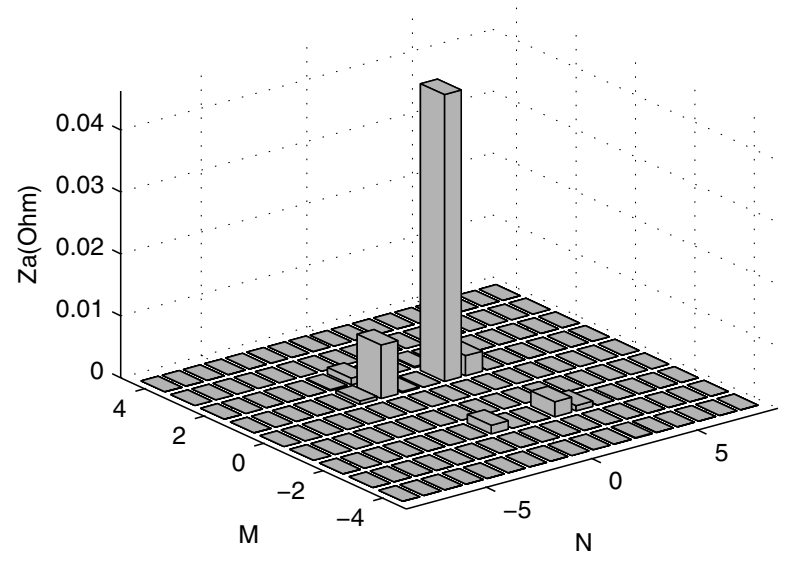

Figure 7. Partial spectral contributions to the antenna module impedance $Z_{a}$. The generator frequency is $f=2.3 \mathrm{MHz}, \mathrm{n}_{0}=$ $3 \times 10^{19} \mathrm{~m}^{-3}$.

The analysis of the radial distribution of the $R F$ power input shows that the main part of the RF power is deposited 
in the regions, which are close to plasma boundary. The radial localization of the $R F$ power input zones for this case is shown in Fig. 8.

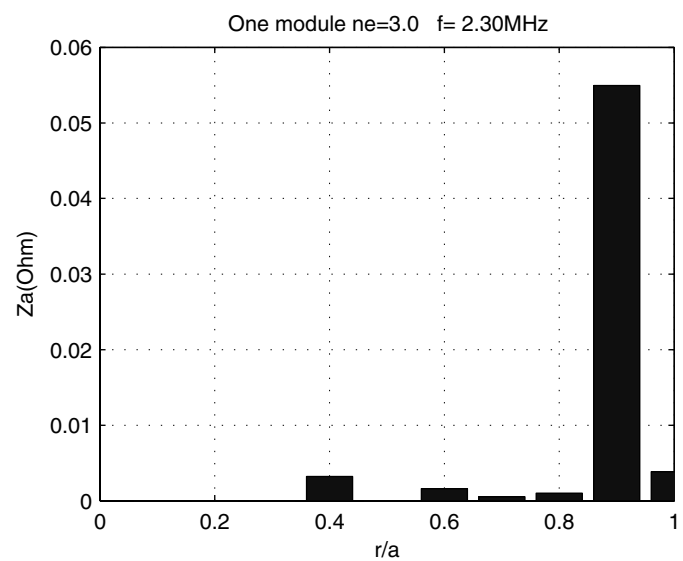

Figure 8. Dependence of antenna module partial impedance on the plasma radius, $f=2.3 \mathrm{MHz}, n_{0}=3 \times 10^{19} \mathrm{~m}^{-3}$.

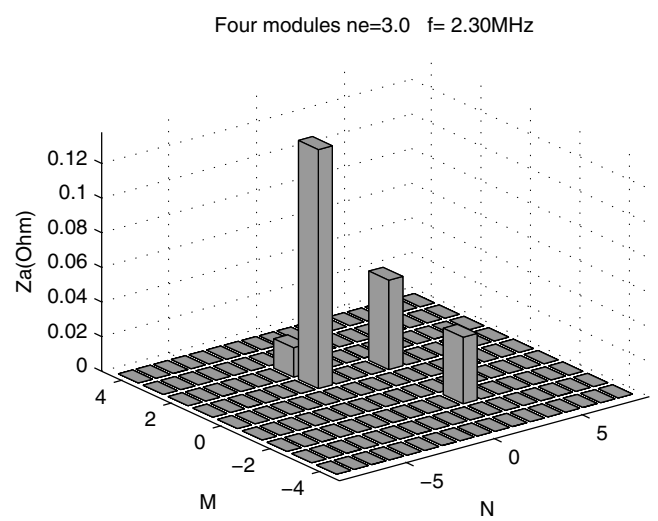

Figure 9. Partial spectral contributions to the antenna module impedance $Z_{a}$. The generator frequency is $f=2.3 \mathrm{MHz}, \mathrm{n}_{0}=$ $3 \times 10^{19} \mathrm{~m}^{-3}$. The antenna consists of four modules.

It is seen that the contribution of the mode $M=-1$, $N=-1$ defines the total antenna impedance and makes it impossible to control the localization of the $R F$ power input in the predefined plasma region.

This situation can be improved if more antenna modules are activated. It has to be mentioned here that the AW excitation has a coherent nature, which makes it different, for example, from the electron cyclotron wave excitation. It means that the field amplitudes, which are excited by the all antennae, are summed, and that is why the activating of the additional antenna modules increases the antenna impedance as well. The spectrum distribution of the antenna impedance in the case, when all four modules are activated, is given in Fig. 9. The phasing of the feeding current was optimized in order to excite predominantly the mode $M=-1$, $N=-2$.
It is seen that the $R F$ mode selection is better now, but nevertheless many additional modes are excited as well. The corresponding radial distribution of the RF power input is shown in Fig. 10. It is seen that the portion of the $R F$ power input in the inner part of the plasma column is higher now, but the RF power input at the plasma boundary is still high.

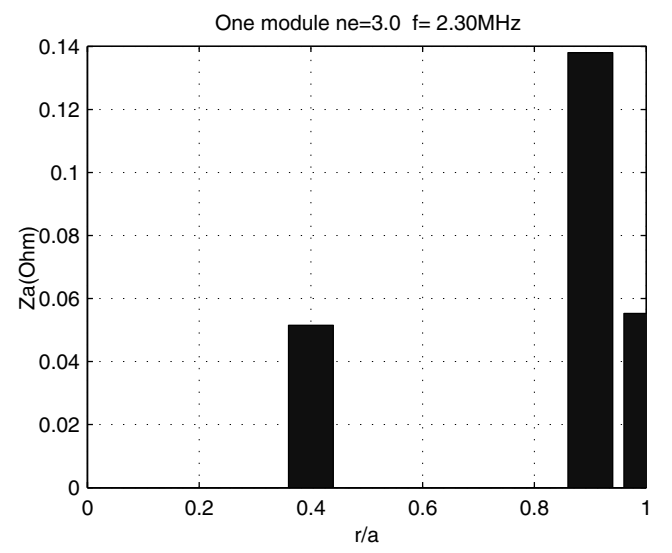

Figure 10. Dependence of the antenna module impedance on the plasma radius, $f=2.3 \mathrm{MHz}, n_{0}=3 \times 10^{19} \mathrm{~m}^{-3}$. Four antenna modules are activated.

The profound changes in the possibility to control the localization of the RF deposition zone occur when the frequency is decreased, and the mode $\mathrm{M}=1, \mathrm{~N}=1$ becomes principal. The spectrum distribution of the antenna impedance in this case is given in Fig. 11. It is seen that the main part of the antenna impedance is due to the excitation of the AW mode $M=1, N=1$.

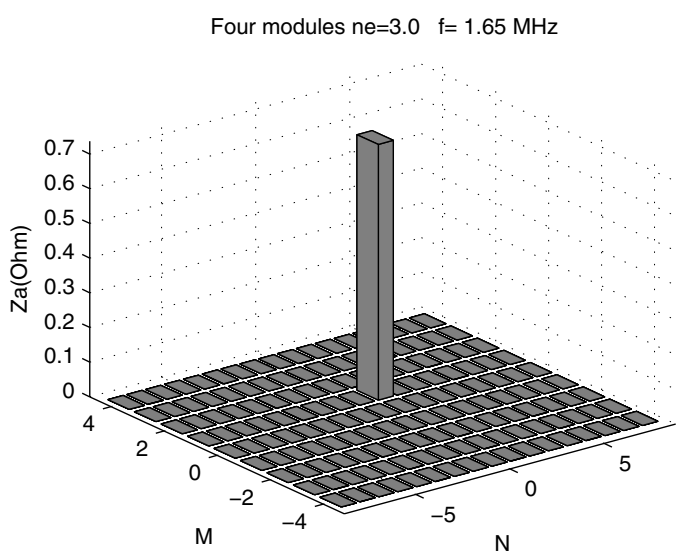

Figure 11. Partial spectral contributions to the antenna module impedance $Z_{a}$. The generator frequency is $f=1.65 \mathrm{MHz}$, $n_{0}=3 \times 10^{19} \mathrm{~m}^{-3}$. The antenna consists of the four modules. The phasing of antenna feeding currents corresponds to the excitation of the AW mode $M=1, N=1$. 


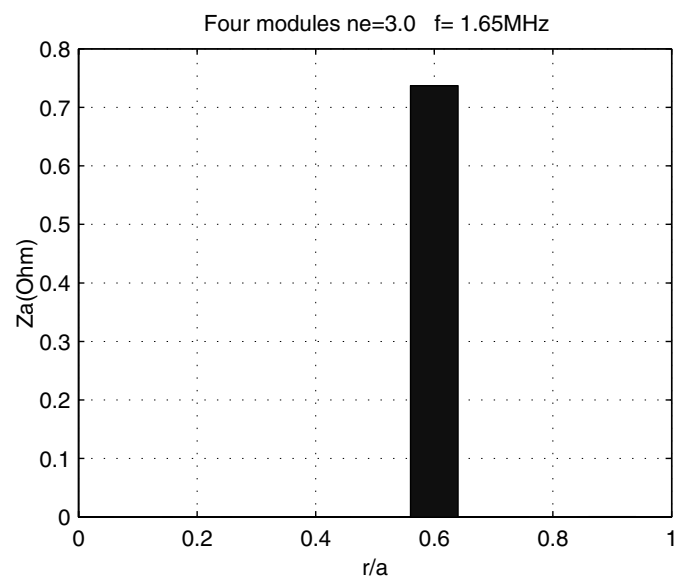

Figure 12. Dependence of the antenna module impedance on the plasma radius, $f=1.65 \mathrm{MHz}, n_{0}=3 \times 10^{19} \mathrm{~m}^{-3}$. Four antenna modules are activated.

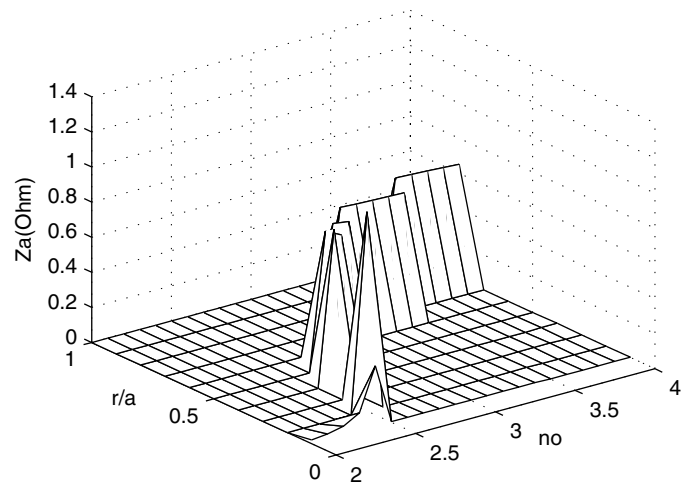

Figure 13. Radial dependence of the $R F$ power input localization on the plasma central density. The antennae are formed by four modules. The $R F$ generator frequency is $1.65 \mathrm{MHz}$.

The corresponding radial distribution of the RF power input is shown in Fig. 12.

It is seen that the localization of the power deposition area is completely controlled by the AW system.

The dependence of the RF power input localization on the plasma density for this case is shown in Fig. 13. It is seen that the area of the RF power input is well controlled both by the AW system regime and by the plasma discharge parameters.

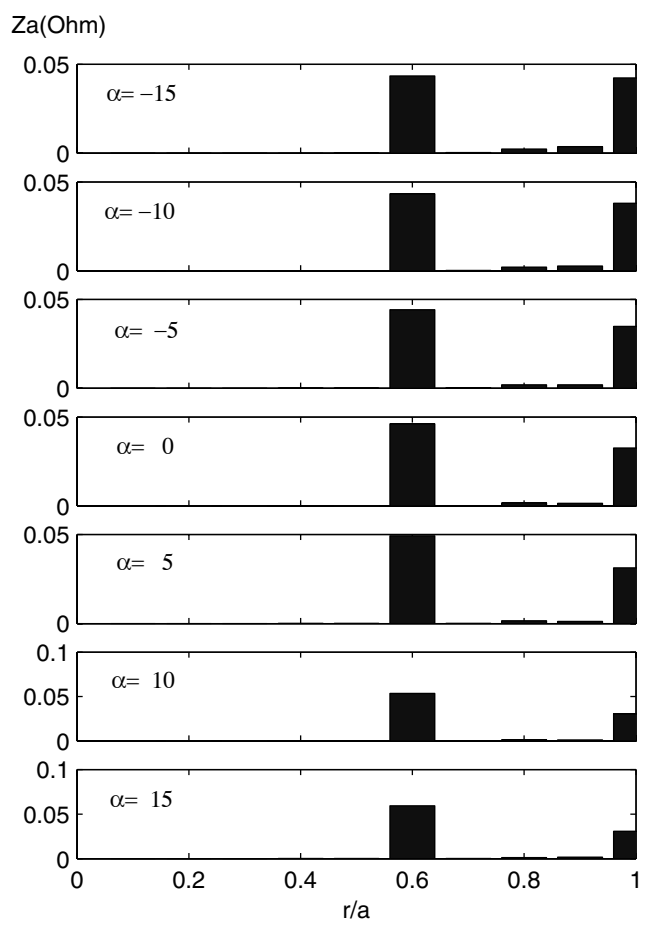

Figure 14. Dependence of the radial distribution of the $R F$ power input on the inclination angle $\alpha$ of the antenna straps. The angular magnitude of $\alpha$ is given in degrees.

The further improvements in the selectivity of the AW mode excitation and in the increasing of the antenna-plasma coupling can be obtained by an inclination of antenna straps in the toroidal direction, as is shown in Fig. 6. The calculated dependence of the radial distribution of the RF power input on the inclination angle $\alpha$ is shown in Fig. 14. It is seen that the antenna strap inclination in a rather narrow range of angles $\left(\alpha=10-15^{\circ}\right)$ permits to redistribute the $R F$ power input between the different AW modes, to decrease the power input at the plasma periphery, and to increase the antenna impedance. The design of the AW antenna for the ETE spherical tokamak provides the possibility to carry out the experiments on the comparative study of different antenna configurations and inclination angles.

\section{Conclusion}

The RF system of the ETE tokamak was designed with the objective to ensure both the effective plasma heating and the non-inductive current drive. In the course of the RF system design the results of the experiments on the Alfvén wave heating and current drive, which were carried out in the TCABR tokamak, were used. The proposed RF system incorporates components and technical solutions, which were tested and approved in the course of the design and examination of the RF system of the TCABR tokamak.

In the course of the simulations the attention was given to the finding the best conditions for the $R F$ power deposition in the plasma inner regions. The effects of the number 
of antenna straps, of the antenna feeding phasing and of the antenna inclination angles were studied.

The analysis of the AW antenna system implementation for the ETE spherical tokamak, which is presented in this work, has to be considered as the first preliminary step for the more detailed future design. The physical processes, which accompany the AW excitation in the spherical tokamak, are much more complicated, and have to be analyzed in 2-D computational models. This work outlines only the questions, which have to be analyzed more thoroughly later.

\section{Acknowledgments}

The authors wish to acknowledge helpful discussions with G. O. Ludwig, E. Del Bosco, and A. G. Elfimov. This work has been supported by The National Council for Scientific and Technological Development CNPq, and The State of São Paulo Research Foundation FAPESP.

\section{References}

[1] J. R. Wilson, R. E. Bell, S. Bernabei M. Bitter, P. Bonoli, D. Gates, J. Hosea, B. LeBlanc, T.K. Mau, S. Medley, J. Menard, D. Mueller, M. Ono, C.K. Phillips, R.I. Pinsker, R. Raman, A. Rosenberg, P. Ryan, S. Sabbagh, D. Stutman, D. Swain, Y. Takase, and J. Wilgen, Phys. Plasmas 10, 1733 (2003).

[2] S. Cuperman, C. Bruma, and K. Komoshvili, Phys. Letters A 311, 221 (2003).

[3] A. Hasegava and L. Chen, Phys. Fluids 19, 1924 (1976).

[4] A. G. Elfimov, 2nd Joint Grenoble-Varenna Int. Symp. on Heating in Toroidal Plasmas, Como, Italy, 683, (1980).
[5] D. W.Ross, G. L.Chen and S. M. Mahajan Phys. Fluids, 25, 652 (1982).

[6] K. Appert, R. Gruber, F. Troyon, and J. Vaclavik, Plasma Phys. 24, 1147 (1982).

[7] K. Appert, G.A. Collins, F. Hofmann, R. Keller, A. Lietti, J.B. Lister, A. Pochelon, and L. Villard, Phys. Rev. Lett., 2, 1671 (1985).

[8] J. Vaclavik and K. Appert, Nucl. Fusion, 31, 1945 (1991).

[9] G. Amarante-Segundo, A. G. Elfimov, D. W. Ross, R. M.O. Galvo, and I. C. Nascimento, Phys. Plasmas, 6, 2437 (1999).

[10] S. A.Galkin, A. A. Ivanov, S. Yu. Medvedev, and A. G. Elfimov, Comp. Phys. Communications, 143/1, 29 (2002).

[11] K. G. McClements, L. C. Appel, M. J. Hole, and A. Thyagaraja, Nucl. Fusion 42, 1155 (2002).

[12] L. F. Ruchko, M. C. Andrade, R.M.O.Galvão, Nuclear Fusion 36, 503 (1996).

[13] K. Appert, J. Vaclavic, and L. Villard. Introduction to the theory of Alfvén wave heating. Lausanne, CRPP, report LRP 238/84, 1984.

[14] D. W. Ross, Y. Li, S. M. Mahajan, R. B.Michie, Nuclear Fusion 26, 139 (1986).

[15] T. C. Hender, S. J. Allfrey, R. Akers, L.C. Appel, M.K. Bevir, R.J Buttery, M. Gryaznevich, I. Jenkins, O.J. Kwon, K.G. McClements, R. Martin, S. Medvedev, M.P.S. Nightingale, C. Ribeiro, C.M. Roach, D.C. Robinson, S.E. Sharapov, S. Sykes, L. Villard, and M.J. Walsh, Phys. Plasmas, 6, 1958 (1999).

[16] V. K. Gusev, F. Alladio, and A. W. Morris, Plasma Phys. Control. Fusion 45, A59 (2003). 